\title{
LES DISTRIBUTEURS D'ENGRAIS POUR ÉTANGS
}

\author{
Par M. AIFRED MEISTER \\ líreisforster it Oberludwigstorf (Silésie).
}

Les recherches modernes en matière de chimie et d'hydrobiologie ont unis en évidence et précisé les relations qui existent entre la productivité des étangs et la qualité de leurs eaux.

Sous la condition primordiale d'une aécation suffisante, ces dernières sont bonnes pour la pisciculture quand leur réaction est neutre ou légèrement alcaline, c'est-à-dire quand la concentration ou puissance des ionslygdrogrène $(p I)$ est ézrale ou supérieure à 7 ( 1 ).

Mais il est fréfuent yu elle soit inférieure, la présence d'acides minéraus on organiques rendant les eaux médiocres ou mauvaises pour l'élevage du Poisson. C'est le cas normal en terrain silicieux ou tourbeux, ou encore à l'intéricur des massif́s boisés.

Pour tirer alors parti des étangs, il faut neul raliser les acides nuisibles. On y parvient par addition de substances basiques, notamment de chaux. Ces amendements sont aussi néecssaires pour les caux servant à la pisciculture que pour les prairies vì pîlure le bélail. Il y a méme lieu de sétonner que leur utilité ait été reconnue seulement à une cipeque récente.

Anjourthui la terhnique de l'emploi des engrais a elé mise au point par de nombreux travanx, entre lespuels il comvient de mentionner spécialement ceus du Docteur E. Waran, a la station basaroise de Wielenbach (2). Appliquée dans toutes les exploitalions rarpicules bien gérééc, cette pratique prend lonjours plus deslemsion.

Mais, jusqu ici, on s'est bormé a épandre à la main la chaux en poudre ou les antres matières fertilisantes. Dans ces conditions, l'opération, sauf

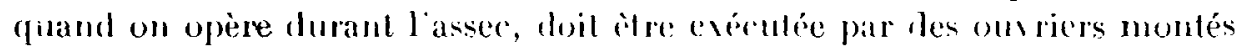
sur barques, elle est alors fort onéreuse el laisse beancoup à désirer, car la répartition des engrais est des plus incigales.

Le recours au machinisme était indiqué pour parer à ce double inconvénient. Je me suis attaché à l'élude de cette question avec la préoccupation de mettre à la disposition des exploitants d'étangs des appareils simples et robustes, à fonctionnenent réxulier et f́conomique.

Je crois y être parvenu en créant deux types de distributeurs d engrais, cntièrement mélalliques, l'un fixe, l'autre mobile.

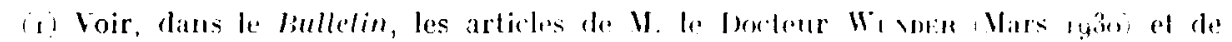

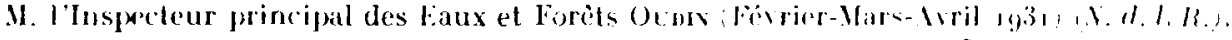

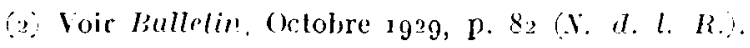

Article available at http://www.kmae-journal.org or http://dx.doi.org/10.1051/kmae:1931014 
Le premier, dénommé Düngumgsmaschine, s’inslalle à demeure aux arrivées d'eau.

Il est composé d’un petit moleur hỵdraulique el de lépandeur.

Le moteur (Figr. I I) est constitué par une roue en dessous, montée sur billes, de 1 mètre de diamètre (1). Il laul, pour l'actionner, aménager ane pelite chute à la queue de l'étangr et dériver sur le coursier un courant d'environ un litre-seconde.

La chaux finement moulue, - on tout autre engrais de mème consisfance, - est versée dans le coffre de l'épandeur qui peut en contenir une

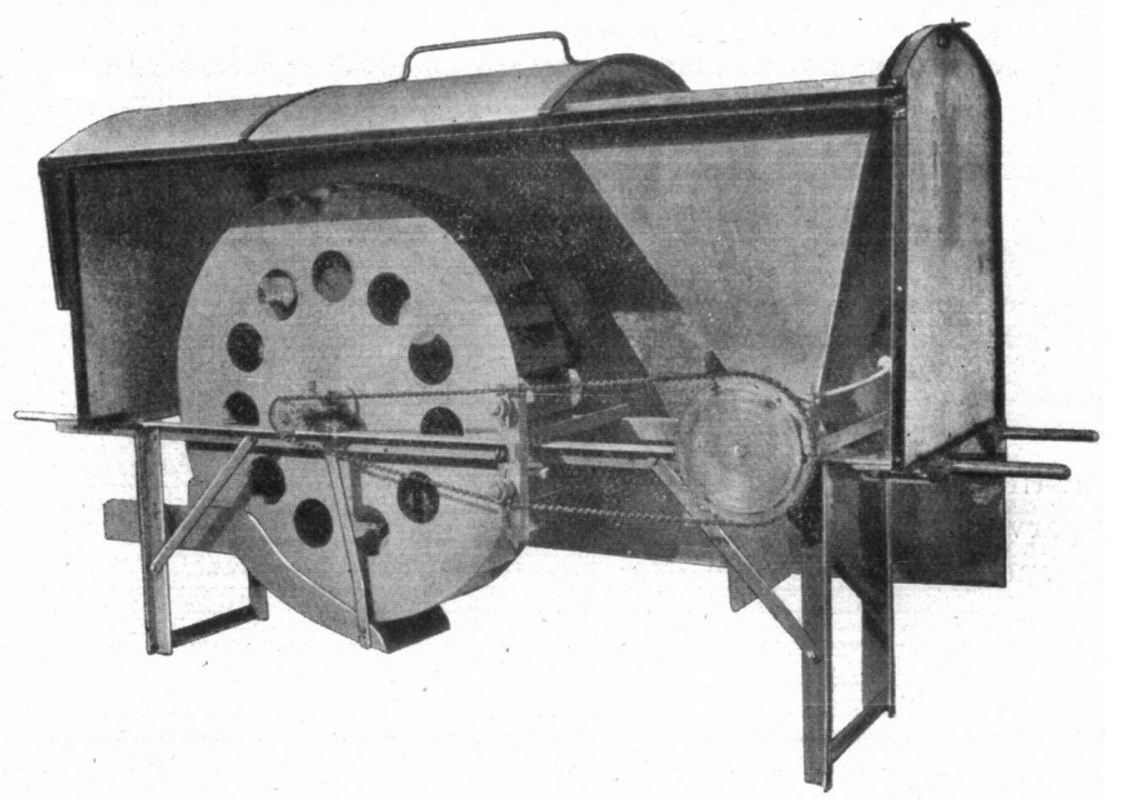

Fir. 11. - Hiingungsmaschine.

centaine de kilogrammes. A sa partic inférieure est une fente devant laquelle coulisse une plaque métallique perforée. Un mouvement de va-etvient lui est imprimé par un arbre horizonfal que la roue motrice entraìne par l'intermédiaire d'une transmission à chaine de Galle et pignons dentés, analogue à celle d'une bicyclette. Ce mème arbre commande un mélangeur, à l’intérieur du coffre, dont le rôle est de brasser constamment la masse pulvérulente pour éviter qu'elle ne s'agglutine. De la surte, elle tombe régulièrement, grâce au déplacement altematif de la coulisse de distribution, sur une grlissière inclinće qui la déverse dans le chenal amenant l'éau à l'étancr.

In couvercle en tôle galvaniséc recouvre tout le distributeur dont al n'y a plus à s'occuper après l'avoir approvisionné.

La consommation de chaux varie ordinairement de jo à roo kilo-

(1) Là où on disjose d'une chute suffisanle, on peut adapter à l'appareil une roue en dessus. 
zrammes par semaine, aver déponse correspontante de z fr. jo à ij francs. mais on peut laugmenter considérablencent, sil est besoin. Le débit de l'appareil dépend de la vitesse de la roue à aubes qu on règlè à volonté. Plus la rotation est rapide, plus on neutralise l'acidité.

Les dimensions de la lsängungsmaschiur sont : - 2 m. 5j en longueur ; - o m. jo en largeur ; - i m. 35 en hauteur ; clle pèse approximativeroment 230 kilogrammes.

Ce distributeur fixe a elé spéciale ment établi pour améliorer la condition des étangs situés en forèt qui, dans la rè̀gle, reçoivent des eaux charcrées d'acides oraranirpues empruntés à la comerture morte du sol boisó. Il a été

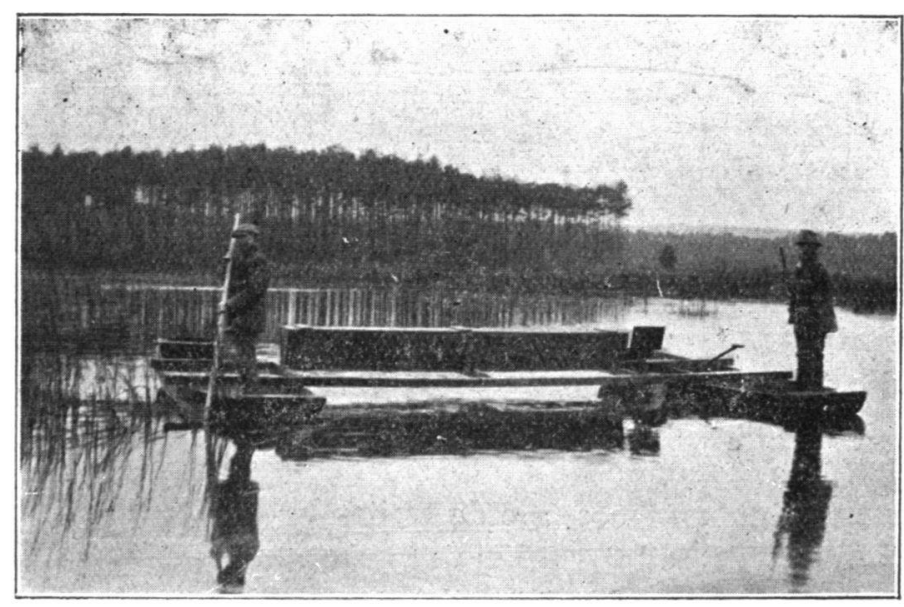

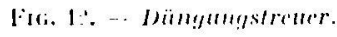

présenté en fonctionnoment, an mois de Juin demier, aux étangrs de Kauppa, près de Bautzen, à la suite d’une conférence du Docteur Wohloesutu sur "La théorie et la pratique de la culture du sol des étangs ". La tournée avait éte organiscé par fos I nions piscicoles d'tllemagne et de Saxe (I)eutscher Fischereivercin ot Sichsischer Fischereiverein); le compte-rendu a fait ressortir que l'appareil que je venais de faire construire se recommandail de lui-mine, comme apportant une solution brillante au problème de l'alcalinisalion des eaux à pH insuffisant (r).

Depuis lors, j’ai imatriné of réalisé un second modèle répondant à un autre besoin, celui de l'épandagre en trand à la surface des élangs.

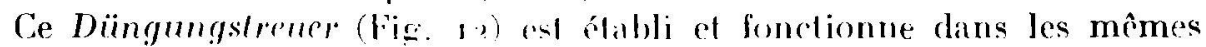
conditions que lo type précédent, mais an are lesodifications qua imposées l'obligration de le rendre tranpontable ol daugrmenter considérablement la largeur d'épandiage.

Le coffe devient ainsi une sorte dituge, longue de mètres, large de r m. İ et haute senlement de a m. for. Elle repose sur un hàti constitue

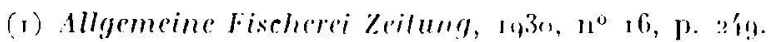


par deux longerons de $\overline{\mathbf{5}} \mathrm{m}$. 40 réunis par trois entretoises dont les extrémités reposent sur deux embarcations, les maintenant parallèles et à ecartement constant.

L'arbre qui commande le mélangeur et la coulisse de distribution se ment lorsque res harques se déplacent, sous l'action de palettes calées ì chacune de ses extrémités (1). Il est monté sur billes.

Le fonctionnement n'a pas besoin d'ère amplement exposé. Les barques se manoureut à la perche ct regoisent la provision d'engrais nécessaire jour l'alimentation du colfie durant toute la séance de travail projeté. On peut aussi, avantageusement, faire remorquer lensemble par un canot automolile, micux encorc par un faucardeur, en effectuant simultanément, quand la chose est possible, la coupe des Jones ou Roseaux et le chaulage.

Quant au profit réalisé par utilisation du distributeur mobile, il est evident. Dienc part, on fait une sérieuse économie de main-d'ouvre. Fn cutre, lorsquion opère à la main, la dissémination est défectueuse, les niatières neutralisantes ou fertilisantes, au contact de l'cau, s'agglomèrent et tombent sur fond par paquets pous y former des amas qui ne se délitent pas. Au contraire, avec le Düngungstreuer, c'est une matière finement divisée qui est déversée en nappe dans une eau qu'agitent le mouvement les hatcaux et celui des palettes ; cet épandage automatique procure, à résultal égal, une économie de moitié sur l'amendement.

Il est donc maintenant possible de traiter les étangs ne se prêtant pas à İinstallation d'un aparcil fixe à l'arrivée d'eau.

Nais, même pour ces derniers, il est possible d'utiliser le sccond at récent modèle pour effectuer une ncutralisation d'ensemble au printemps, ce après quoi, jusqu'à la fin de la belle saison, on maintient l'étang en honne condition en désacidifiant, au fur et à mesure de son admission, l'eau d'alimentation.

Les carpiculteurs ont donc maintenant à leur disposition, pour une exploitation rationnelle, des distributeurs d'engrais similaires de ceux uilisés pour l'amendement. des terres. Ceux que j’ai imaginés ont donné satisfaction en Allemagne où l'usage commence à s'en répandre ; j'ai lieu d'espérer qu'ils pourront aussi être appréciés en France et dans les autres pays d'étangs.

(3) Sur la figure il n'existe re paleltes que sur la droite de l'auge, mais il est préférable qu'il y en ait de chaque coté.

Erralum. -- Dans l'article de M. le Professeur Rössı.rs, publiś en Juillet (pages ı 3 i $r$ ), il convient de substituer a l'expression " vase de pheherie " celle de "vase de vucreries ". Il s'agit du dépôt qui se produit dius les hassius de décantation. 